\title{
Light Cell
}

National Cancer Institute

\section{Source}

National Cancer Institute. Light Cell. NCI Thesaurus. Code C13148.

A cell located in the collecting tube of the nephron.. 